Review

\title{
Tutorial: Current Status of Standardization for Practical Surface Analysis Methods and Activities of KRISS
}

\author{
Kyung Joong Kim* \\ Division of Industrial Metrology, Korea Research Institute of Standards and Science, \\ 267 Gajeong-ro, Yusong, Daejeon 305-600, Korea \\ *kjkim@kriss.re.kr
}

(Received : July 15, 2015; Accepted : August 31, 2015)

\begin{abstract}
Surface analysis methods are widely used during the fabrication process of advanced devices. For practical applications in advanced industries, surface analysis methods should be standardized by the establishment of traceability and with the development of measurement procedures and certified reference materials. Measurement traceability for surface analysis is established by key comparisons (KCs) by the Surface Analysis Working Group (SAWG) of the Consultative Committee for Amount of Substance (CCQM). Thus far, two KCs for thickness measurements of $\mathrm{nm} \mathrm{SiO}_{2}$ films (K-32) and for analyses of the atomic fractions of Fe-Ni alloy films (K-67) have been performed. International standards for surface chemical analysis procedures have been developed by ISO/TC-201. Fifty nine international standards have been published. The Korea Research Institute of Standards and Science (KRISS) has participated in key comparisons of the CCQM SAWG as the national metrology institute (NMI) of Korea. KRISS has also participated in ISO/TC-201 to develop international standards for surface analysis using XPS, AES and SIMS. Three types of thin-film certified reference materials have been developed by KRISS.
\end{abstract}

\section{Introduction}

Surface analysis technologies are useful for the fabrication processes of the advanced devices such as semiconductors, displays, solar cells and light-emitting diodes. Surface analysis methods are used to develop useful metrologies for advanced industries, such as quantitative analyses of constituent elements, thickness measurements of thin films, elemental mapping, and depth profiling analyses.

To improve the reliability of the measurement results, measurement issues must be standardized for their practical applications. The establishment of traceability, the development of international standards, and the development of certified reference materials (CRMs) are the key activities for the standardization of surface analysis methods.

Measurement traceability as it pertains to surface analysis is established by the Surface Analysis Working Group (SAWG) of the Consultative Committee for Amount of Substance (CCQM). Key comparisons (KCs) are performed to assess the equivalence of the measurement capabilities of national metrology institutes (NMIs). Measurement issues for key comparisons should be studied beforehand in a pilot study (PS) before the key comparison [1].

The Korea Research Institute of Standards and Science (KRISS) has participated in CCQM SAWG $\mathrm{KCs}$, focused on the establishment of traceability of surface chemical analysis methods related to industrial metrologies. KRISS participated in the pilot study P-38 and the key comparison K-32 for the thickness measurement of $\mathrm{nm} \mathrm{SiO}$ films [2-4]. KRISS organized the key comparison K-67 for the analysis of the atomic fractions of $\mathrm{Fe}-\mathrm{Ni}$ alloy films [5,6]. KRISS has participated in ISO/TC-201 as a $\mathrm{P}$ member and has contributed to the development of international standards. Many types of thin-film CRMs have been developed by KRISS.

In this paper, the key activities of KRISS for the standardization of practical surface analysis methods are reviewed with regard to the establishment of measure- 
ment traceability, the development of international standards and the fabrication of CRMs.

\section{Establishment of Traceability}

The improvement of world-wide traceability of measurement results is the unique role of the Bureau International des Poids et Mesures (BIPM) in Paris, France. The traceability of measurements is established by consultative committees of the International Committee for Weights and Measures (CIPM) via mutual recognition arrangements between NMIs to demonstrate the international equivalence of their measurement standards [1].

The final outcomes of the consultative committees of CIPM are the internationally recognized calibration and measurement capabilities (CMCs) of the participating institutes. Approved CMCs and supporting technical data are available from the CIPM MRA database (KCDB).

In CIPM, ten consultative committees have been organized for metrological areas, as shown in Table 1. Metrology in chemistry and biology is investigated by the CCQM. Seven working groups have been organized under CCQM, as shown in Table 2 [7].

The measurement traceability of surface analysis methods is investigated by CCQM SAWG. KCs to compare the equivalence of measurement capabilities by NMIs and the resultant claims of CMCs are the main procedures by which to establish measurement traceability. Thus far, two key comparisons and eight pilot studies have been completed by CCQM SAWG, as shown in Table 3.

Table 1. Metrological areas of the consultative committees in CIPM.

\begin{tabular}{ll}
\hline Name & Metrology Area \\
\hline CCL & Length \\
CCM & Mass and Related Quantities \\
CCTF & Time and Frequency \\
CCT & Thermometry \\
CCPR & Photometry and Radiometry \\
CCEM & Electricity and Magnetism \\
CCQM & Amount of Substance \\
CCRI & Ionizing Radiation \\
CCAUV & Acoustics, Ultrasound and Vibration \\
CCU & Units \\
\hline
\end{tabular}

Table 2. Metrological areas of the working groups of CCQM.

\begin{tabular}{ll}
\hline Name & Metrology Area \\
\hline KCWG & Key Comparison \\
GAWG & Gas Analysis \\
EAWG & Electric Analysis \\
IAWG & Inorganic Analysis \\
OAWG & Organic Analysis \\
BAWG & Bio Analysis \\
SAWG & Surface Analysis \\
\hline
\end{tabular}

The pilot studies P-84 and P-108 were performed in parallel with key comparisons K-32 and K-67, respectively. Recently, P-140 and K-129 were performed for the quantitative analysis of $\mathrm{Cu}(\mathrm{In}, \mathrm{Ga}) \mathrm{Se}_{2}$ films as an example of a multi-element alloy film [8].

\subsection{Thickness measurement of $\mathrm{SiO}_{2}$ film}

Thickness measurement of gate oxide films thinner than $1 \mathrm{~nm}$ equivalent thickness is one of the most important metrology issues for next- generation semiconductor devices. For this reason, measuring the thickness of $\mathrm{nm} \mathrm{SiO}_{2}$ films was selected as the first subject of CCQM SAWG. The pilot study P-38 and key comparison K-32 were organized by NPL for the thickness of $\mathrm{SiO}_{2}$ on $\mathrm{Si}(100)$ and $\mathrm{Si}$ (111) substrates with a nominal thickness range of 1.5 to $8 \mathrm{~nm}$.

In P-38, many measurement methods, such as spectroscopic ellipsometry (SE), transmission electron mi-

Table 3. Completed key comparisons and pilot studies by CCQM SAWG.

\begin{tabular}{lll}
\hline No. & Description & $\begin{array}{l}\text { Start } \\
\text { year }\end{array}$ \\
\hline P-38 & $\mathrm{SiO}_{2}$ on Si film thickness & 2002 \\
\hline P-80 & Carbon in precipitates in Fe & 2005 \\
\hline P-81 & N in surface layers of Fe & 2005 \\
\hline K-32 & $\mathrm{SiO}_{2}$ on Si film thickness & 2005 \\
\hline P-84 & $\mathrm{SiO}_{2}$ on Si, surface analysis & 2005 \\
\hline P-95 & $\mathrm{Nitrogen} \mathrm{content} \mathrm{in} \mathrm{DLC} \mathrm{layers}$ & 2006 \\
\hline P-98 & $\mathrm{Composition} \mathrm{of} \mathrm{Fe-Ni} \mathrm{Alloy} \mathrm{films}$ & 2007 \\
\hline K-67 & Quantitative analysis of Fe-Ni alloy & 2008 \\
\hline P-108 & Quantitative analysis of Fe-Ni alloy & 2008 \\
\hline P-140 & CIGS layer composition & 2013 \\
\hline
\end{tabular}


croscopy (TEM), Rutherford backscattering spectrometry (RBS), grazing incidence $\mathrm{x}$-ray reflectometry (GIXRR), nuclear reaction analysis (NRA), medium energy ion scattering (MEIS), neutron reflectometry (NR), and X-ray photoelectron spectroscopy (XPS), were compared [2].

The thickness of $\mathrm{SiO}_{2}$ films $\left(T_{o x}\right)$ on $\mathrm{Si}$ substrates is determined from the Si $2 p$ core-level spectrum by the following equation.

$$
T_{o x}=L \cos \theta \ln \left(R_{\text {exp }} / R_{0}+1\right)
$$

Here, $L$ is the effective attenuation length of $\mathrm{Si} 2 \mathrm{p}$ in the $\mathrm{SiO}_{2}$ matrix and $\theta$ is the angle between the surface normal and the analyser. $R_{0}\left(=\mathrm{I}_{\mathrm{SiO} 2} / \mathrm{I}_{\mathrm{Si}}{ }^{\infty}\right)$ indicates the relative peak intensities of $\mathrm{Si} 2 \mathrm{p}$ as measured from pure $\mathrm{SiO}_{2}$ and pure Si. $R_{\text {exp }}\left(=\mathrm{I}_{\mathrm{SiO} 2}{ }^{\text {exp }} / \mathrm{I}_{\mathrm{Si}}{ }^{\text {exp }}\right)$ denotes the relative peak intensity of $\mathrm{Si} 2 \mathrm{p}$ as measured from a $\mathrm{SiO}_{2}$ overlayer on $\mathrm{Si}$.

If $L, \theta$ and $R_{0}$ are determined correctly by experiments or theoretical calculations, the thickness can be derived from the measurement of the $R_{\text {exp }}$ value.

The important result of P-38 is that the thickness results by SE, TEM, RBS, XRR, NRA, MEIS and NR showed large offset values ranging from $0.2 \mathrm{~nm}$ to 1.0 $\mathrm{nm}$. However, the thickness results by XPS showed a small offset value close to zero because the thickness of $\mathrm{SiO}_{2}$ film by XPS is determined from the peak intensity of the $\mathrm{SiO}_{2}$ component $\mathrm{Si} 2 \mathrm{p}$ spectra.

On the basis of P-38, key comparison K-32 was also organized by NPL. Nine NMIs (NPL, NRCCRM, CSIR, BAM, NIST, KRISS, NIMT, PTB and NMIJ) participated in K-32 using XPS, NR, XRR and SE. Five $\mathrm{SiO}_{2}$ films with nominal thicknesses 1.5, 2, 3, 4, and $8 \mathrm{~nm}$ on $\mathrm{Si} \mathrm{(100)} \mathrm{and} \mathrm{four} \mathrm{SiO}_{2}$ films with nominal thicknesses 2, 3, 5 and $6 \mathrm{~nm}$ on $\mathrm{Si}$ (111) were used. The (100) Si wafers were cut into squares and the Si (111) wafers were cut into triangles. The homogeneity of the oxide thickness mapped by a spectroscopic ellipsometer showed a precision level of around $0.002 \mathrm{~nm}$.

The thicknesses measured by XPS in K-32 showed strong equivalence to the key comparison reference value with a low level of uncertainty. To improve the level of uncertainty during the thickness measurements of oxide films, KRISS developed a mutual calibration method to determine the effective attenuation length [9] and an overlayer thickness consistency method to determine the surface normal for the precise determination of the electron emission angle [10].

Recently, CCQM agreed to the creation of a new policy for the repetition of important key comparisons. As a result, CCQM SAWG is preparing a new key comparison to repeat K-32 with regard to the thickness measurements of $\mathrm{SiO}_{2}$ or $\mathrm{HfO}_{2}$ films.

\subsection{Quantification of Fe-Ni alloy Films}

A quantitative surface analysis of alloy films by XPS and AES is one of the most useful applications of surface analysis methods [11]. However, a quantitative surface compositional analysis of a multi-component system is complicated due to the matrix effect and surface composition changes caused by sputtering with an ion beam. An ideal solution for the quantitative analysis of binary alloy films is to use an alloy reference film with a composition close to the analysis specimen. In this case, the matrix effect and preferential sputtering are counterbalanced between a reference and a sample to be measured [12].

The CCQM pilot study P-98 and the key comparison K-67 were organized by KRISS for the standardization of a quantitative analysis of Fe-Ni alloy film as an example of a simple binary alloy film. Three alloy films with nominal atomic fractions of Fe28-Ni72, Fe51-Ni49 and $\mathrm{Fe} 78-\mathrm{Ni} 22$ were used as analysis specimens for P-98. The compositions of the alloy films were certified by inductively coupled plasma mass spectrometry with isotope dilution (ID-ICP/MS). The in-depth and lateral homogeneities of the atomic fraction were confirmed by secondary ion mass spectrometry (SIMS) using $\mathrm{C}_{60}$ primary ions. Fe51-Ni49 alloy film was used as a reference specimen to determine the relative sensitivity factors (RSFs) of Fe and Ni [12].

P-98 was performed to investigate the feasibility of measurement equivalency for the atomic fractions of Fe-Ni alloy films. Nine laboratories participated in P-98 using XPS, AES and EPMA. The compositions of the alloy films were measured by two types of sensitivity factors. The first type is pure element RSF (PERSF; $\mathrm{S}_{\mathrm{Fe}}{ }^{\infty}$ and $\mathrm{S}_{\mathrm{Ni}}{ }^{\infty}$ ) derived from pure $\mathrm{Fe}$ and $\mathrm{Ni}$ films. The second type is alloy reference RSF (ARRSF) which is determined from the certified atomic fractions of the reference film. The ARRSFs of $\mathrm{Fe}\left(\mathrm{R}_{\mathrm{Fe}}{ }^{\mathrm{AR}}\right)$ and $\mathrm{Ni}\left(\mathrm{R}_{\mathrm{Ni}}{ }^{\mathrm{AR}}\right)$ were derived from a certified Fe51-Ni49 alloy film by dividing the peak intensities $\mathrm{Fe}\left(\mathrm{I}_{\mathrm{Fe}}{ }^{\mathrm{AR}}\right)$ and $\mathrm{Ni}\left(\mathrm{I}_{\mathrm{Ni}}{ }^{\mathrm{AR}}\right)$ by the 


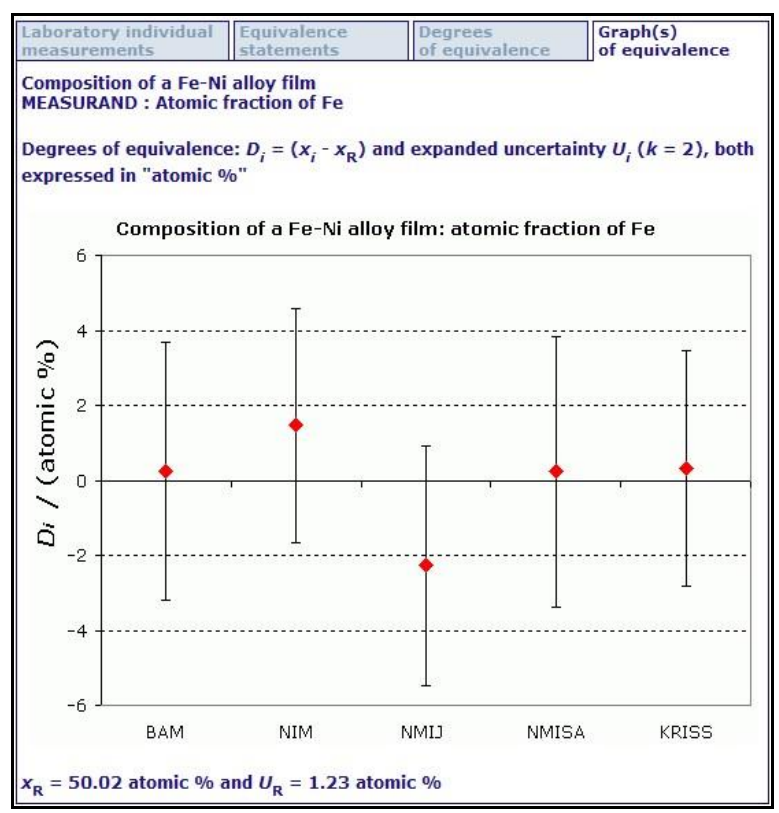

Figure 1. Degrees of equivalence and expanded uncertainties of CCQM K-67 [1]. (color online)

certified atomic fractions of $\mathrm{Fe}\left(\mathrm{C}_{\mathrm{Fe}}{ }^{\mathrm{AR}}\right)$ and $\mathrm{Ni}\left(\mathrm{C}_{\mathrm{Ni}}{ }^{\mathrm{AR}}\right)$, respectively. The measured atomic fractions were linearly fitted as a function of the certified atomic fractions by linear least square fitting with the following equation.

$$
X_{\text {meas }}=m X_{\text {cert }}+c
$$

Here, the offset $\mathrm{c}$ is the excess fraction when the certified atomic fraction is extrapolated to zero and the slope $\mathrm{m}$ is a scaling constant. The ideal values of $\mathrm{m}$ and $\mathrm{c}$ are 1 and 0 , respectively.

Although the average slope does not change, the average offset value was improved from 0.725 to 0.038 using PERSFs and ARRSFs, respectively. This showed that the ARRSFs are much better for equivalency during the quantification of Fe-Ni alloy films than the PERSFs [5].

K-67 involved a quantitative analysis of $\mathrm{Fe}-\mathrm{Ni}$ alloy film. As a result of P-98, the Fe51-Ni49 alloy film was supported as a certified reference specimen to determine the RSFs. The measurand of K-67 was atomic fraction of Fe. Four NMIs (NMIA, KRISS, NIM and NMIJ) and one DI (BAM) participated in K-67. In the quantitative analysis of an alloy film by surface analysis methods using a CRM, the combined standard uncertainty $u_{\mathrm{c}}$ can be determined from the equation $u_{c}^{2}=u_{\text {meas }}^{2}+u_{C R M}^{2}$.

The first term $\left(u_{\text {meas }}\right)$ is the standard uncertainty in the measurement of the atomic fraction of the alloy film. It is derived from a combination of the standard uncertainties in the determinations of RSFs $\left(u_{\mathrm{RSF}}\right)$ and from measurements of the atomic fractions $\left(u_{\mathrm{All}}\right)$ of the alloy film. The second term is the standard uncertainty in the certification of the atomic fraction of the alloy film. The expanded uncertainties in K-67 ranged from 2.88 to 3.40 atomic \%.

The key comparison reference value (KCRV) determined from the arithmetic mean of the submitted values was 50.02 at\%, as shown in Figure 1. The uncertainty of $\mathrm{KCRV}$ as calculated from the standard deviation of the individual reported values and a coverage factor $(\mathrm{k})$ of 2 was 1.23 at\% [6].

\subsection{Quantification of $\mathrm{Cu}(\mathrm{In}, \mathrm{Ga}) \mathrm{Se}_{2}$ films}

$\mathrm{Cu}(\mathrm{In}, \mathrm{Ga}) \mathrm{Se}_{2}$ (CIGS) was selected as a representative multi-element alloy material for a pilot study of quantitative analysis methods by CCQM SAWG in 2013. The CIGS thin film solar cell is a next-generation solar cell with high conversion efficiency and a low fabrication cost. During the fabrication of CIGS solar cells, the relative atomic fractions and in-depth distributions of $\mathrm{Cu}$, In, $\mathrm{Ga}$ and Se are very important to form the chalcopyrite crystal structure for the improvement of the solar conversion efficiency [13-16].

P-140 was performed to investigate the feasibility of
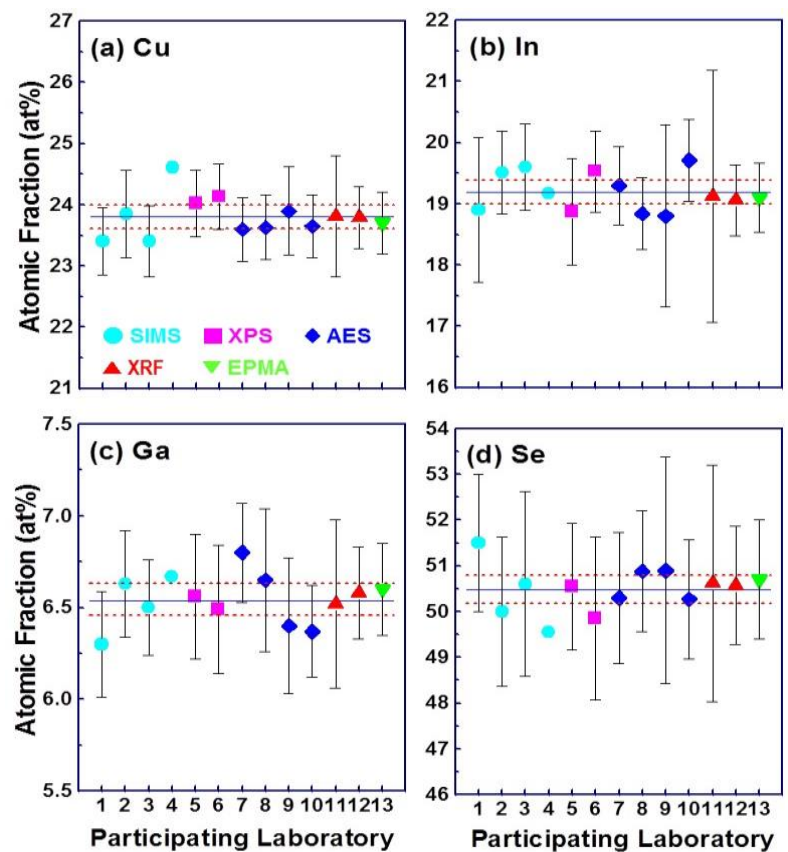

Figure 2. The measured atomic fractions and uncertainties of (a) $\mathrm{Cu}$, (b) In, (c) Ga and (d) Se in a test specimen by SIMS (cyan circle), XPS (magenta square), AES (blue diamond), XRF (red triangle) and EPMA (green downward triangle). (color online) 
the measurement equivalency of the atomic fractions of CIGS films. Fifteen laboratories from eight countries participated in P-140 using SIMS, XPS, AES, XRF and EPMA. The atomic fractions of the four test CIGS films were measured and compared. The total number counting (TNC) method was recommended as a method by which to determine the relative sensitivity factors of the constituent elements using a reference CIGS film certified by ID-ICP/MS [17].

The average mole fractions of the reported data showed relative standard deviations in a range of $5.5 \%$ to $6.8 \%$, with the average relative expanded uncertainties ranging from $4.52 \%$ to $4.86 \%$ for the four test CIGS specimens. These values are smaller than those in K-67 for the measurement of the mole fractions of $\mathrm{Fe}-\mathrm{Ni}$ alloy films. The relative standard deviations were greatly improved to $1.5 \% \sim 2.2 \%$ by excluding five highly deviant data sets. Figure 2 shows the measurement results of a test specimen. The uncertainties of the reference values calculated from the standard deviations of the reported results and the coverage factor of 2.18 are 0.19 , $0.19,0.08$ and 0.30 at\% for $\mathrm{Cu}, \mathrm{In}, \mathrm{Ga}$ and $\mathrm{Se}$, respectively. Although the results were measured by various methods, the variations of the results are not significant.

This result indicates that quantification by depth profiling with the TNC method is reliable for the quantitative analysis of multi-element alloy films and that CIGS film is suitable as a material for a new key comparison.

\section{Development of Standards}

The second parameter of the standardization process is the development of standards. The step-by-step measurement process of an approved traceable method should be documented as a type of international standard for its application.

International standards are developed by the International Organization for Standardization (ISO) founded in 1947 as an independent, non-governmental membership organization. ISO standards are developed from consensus-based negotiations of the members of technical committees (TC) composed of groups of experts from the relevant industry, consumer associations, academia and government. Thus far, more than 19500 standards have been published by approximately 300 TCs [18].

\subsection{ISO/TC-201 (Surface Chemical Analysis)}

International standards in the area of surface chemical analysis are developed by ISO/TC201, which was created in 1991. In 1993, seven subcommittees (SC) were initially created for the investigation of terminology (SC1), general procedures (SC2), data management and treatments (SC3), depth profiling (SC4), AES (SC5), SIMS (SC6) and XPS (SC7). Subcommittees for glow discharge spectroscopy (SC8) and scanning probe microscopy (SC9) were created in 1998 and 2004, respectively. The SC for AES (SC5) was merged into that for XPS (SC7) with the new name of electron spectroscopy (SC7) in 2012. Thus far, 59 international standards have been published from eight subcommittees (SC), one study group (SG) and three working groups (WG), as shown in Table 4 and Table 5.

\subsection{Standard of a Traceable Method}

The standardization of a traceable method approved by a key comparison of CCQM SAWG can be simplified using the measurement protocol of the KC. XPS has been proved as a traceable method for the thickness measurements of $\mathrm{nm} \mathrm{SiO}_{2}$ films on $\mathrm{Si}$ (100) and $\mathrm{Si}$ (111) substrates. The detailed procedure of the thickness measurement of $\mathrm{nm} \mathrm{SiO}_{2}$ films by XPS has been theoretically and practically investigated from pilot study P-38 and key comparison K-32.

Table 4. The research areas of ISO/TC-201 and subcommittees

\begin{tabular}{lll}
\hline No. & Research Area & $\begin{array}{l}\text { No. of } \\
\text { standards }\end{array}$ \\
\hline SC 1 & Terminology & 2 \\
\hline SC 2 & General Procedures & 7 \\
\hline SC 3 & Data Management and Treatment & 4 \\
\hline SC 4 & Depth Profiling & 4 \\
\hline SC 6 & Secondary Ion Mass Spectrometry & 9 \\
\hline SC 7 & Electron Spectroscopy & 19 \\
\hline SC 8 & Glow Discharge Spectroscopy & 5 \\
\hline SC 9 & Scanning Probe Microscopy & 4 \\
\hline SG 1 & Nano-materials Characterization & 0 \\
\hline WG 3 & $\begin{array}{l}\text { X-ray Reflectivity and Total Reflection } \\
\text { X-ray Fluorescence Spectroscopy }\end{array}$ & 4 \\
\hline WG 4 & Surface Characterization of Biomaterials & 0 \\
\hline WG 5 & Optical Interface Analysis & 1 \\
\hline & & \\
\hline
\end{tabular}


Table 5. List of international standards developed by ISO/TC-201 (30 Jun 2015)

ISO-10810: XPS-Guidelines for analysis (SC7)

ISO-11039: SPM-Measurement of drift rate (SC9)

ISO-11505: General procedures for quantitative compositional depth profiling by GD-OES (SC8)

ISO-11952: SPM-Determination of geometric quantities using SPM: Calibration of measuring systems (SC9)

ISO-12406: SIMS-Method for depth profiling of arsenic in silicon (SC6)

ISO-13084: SIMS-Calibration of the mass scale for a TOFSIMS (SC6)

ISO-13095: AFM-Procedure for in situ characterization of AFM probe shank profile used for nanostructure measurements (SC9)

ISO-13424: XPS-Reporting of results of thin-film analysis (SC7)

ISO/TR-14187: Characterization of nanostructured materials (SC7)

ISO-14237: SIMS-Determination of B atomic concentration in silicon using uniformly doped materials (SC6)

ISO-14606: Sputter depth profiling - Optimization using layered systems as reference materials (SC4)

ISO-14701: XPS-Measurement of silicon oxide thickness (SC7)

ISO-14706: Determination of surface elemental contamination on silicon wafers by TXRF spectroscopy (TC201)

ISO-14707: Glow discharge optical emission spectrometry (GD-OES) - Introduction to use (SC8)

ISO-14975: Information formats (SC3)

ISO-14976: Data transfer format (SC3)

ISO-15338: Glow discharge mass spectrometry (GD-MS) - Introduction to use (SC8)

ISO-15470: XPS-Description of selected instrumental performance parameters (SC7)

ISO-15471: AES-Description of selected instrumental performance parameters (SC7)

ISO-15472: XPS-Calibration of energy scales (SC7)

ISO/TR-15969: Depth profiling - Measurement of sputtered depth (SC4)

ISO-16129: XPS-Procedures for assessing day-to-day performance of an X-ray photoelectron spectrometer (SC7)

ISO-16242: Recording and reporting data in AES (SC2)

ISO-16243: Recording and reporting data in XPS (SC2)

ISO/TR-16268: Proposed procedure for certifying the retained areic dose in a working reference material produced by ion implantation (SC2)

ISO-16413: Evaluation of thickness, density and interface width of thin films by XRF - Instrumental requirements, alignment and positioning, data collection, data analysis and reporting (TC201)

ISO-16531: Depth profiling - Methods for ion beam alignment and the associated measurement of current or current density for depth profiling in AES and XPS (SC4)

ISO-16962: Analysis of zinc- and/or aluminium-based metallic coatings by glow-discharge optical-emission spectrometry (SC8)

ISO-17331: Chemical methods for the collection of elements from the surface of silicon wafer working reference materials and their determination by TXRF spectroscopy (TC201)

ISO-17331: Amd1:2010 (TC201)

ISO-17560: SIMS-Method for depth profiling of boron in silicon (SC6)

ISO-17862: SIMS-Linearity of intensity scale in single ion counting time-of-flight mass analysers (SC6)

ISO-17973: Medium-resolution Auger electron spectrometers - Calibration of energy scales for elemental analysis (SC7)

ISO-17974: High-resolution Auger electron spectrometer - Calibration of energy scales for elemental and chemical-state analysis (SC7)

ISO-18114: SIMS-Determination of relative sensitivity factors from ion-implanted reference material (SC6)

ISO-18115-1: Vocabulary-Part 1: General terms and terms used in spectroscopy (SC1)

ISO-18115-2: Vocabulary-Part 2: Terms used in scanning-probe microscopy (SC1)

ISO-18116: Guidelines for preparation and mounting of specimens for analysis (SC2)

ISO-18117: Handling of specimens prior to analysis (SC2)

ISO-18118: XPS and AES-Guide to the use of experimentally determined relative sensitivity factors for the quantitative analysis of homogeneous materials (SC7)

ISO-18337: Surface characterization-Measurement of the lateral resolution of a confocal fluorescence microscope (TC201)

ISO/TR-18392: XPS-Procedures for determining backgrounds (SC7)

ISO/TR-18394: AES-Derivation of chemical information (SC7)

ISO-18516: XPS and AES - Determination of lateral resolution (SC2)

ISO-19318: XPS-Reporting of methods used for charge control and charge correction (SC7)

ISO/TR-19319: Fundamental approaches to determination of lateral resolution and sharpness in beam-based methods (SC2)

ISO-20341: SIMS-Method for estimating depth resolution parameters with multiple delta-layer reference materials (SC6)

ISO-20903: XPS and AES-Methods used to determine peak intensities and information required when reporting results (SC7)

ISO-21270: X-ray photoelectron and Auger electron spectrometers - Linearity of intensity scale (SC7)

ISO-22048: Information format for static secondary-ion mass spectrometry (SC3)

ISO/TR-22335: Depth profiling-Measurement of sputtering rate: mesh-replica method using a mechanical stylus profilometer (SC4)

ISO-23812: SIMS-Method for depth calibration for silicon using multiple delta-layer reference materials (SC6)

ISO-23830: SIMS-Repeatability and constancy of the relative-intensity scale in static SIMS (SC6)

ISO-24236: AES-Repeatability and constancy of intensity scale (SC7)

ISO-24237: XPS-Repeatability and constancy of intensity scale (SC7)

ISO/TR-25138: Analysis of metal oxide films by glow-discharge optical-emission spectrometry (SC8)

ISO-27911: SPM-Definition and calibration of the lateral resolution of a near-field optical microscope (SC9)

ISO-28600: Data transfer format for scanning-probe microscopy (SC3)

ISO-29081: AES-Reporting of methods used for charge control and charge correction (SC7) 


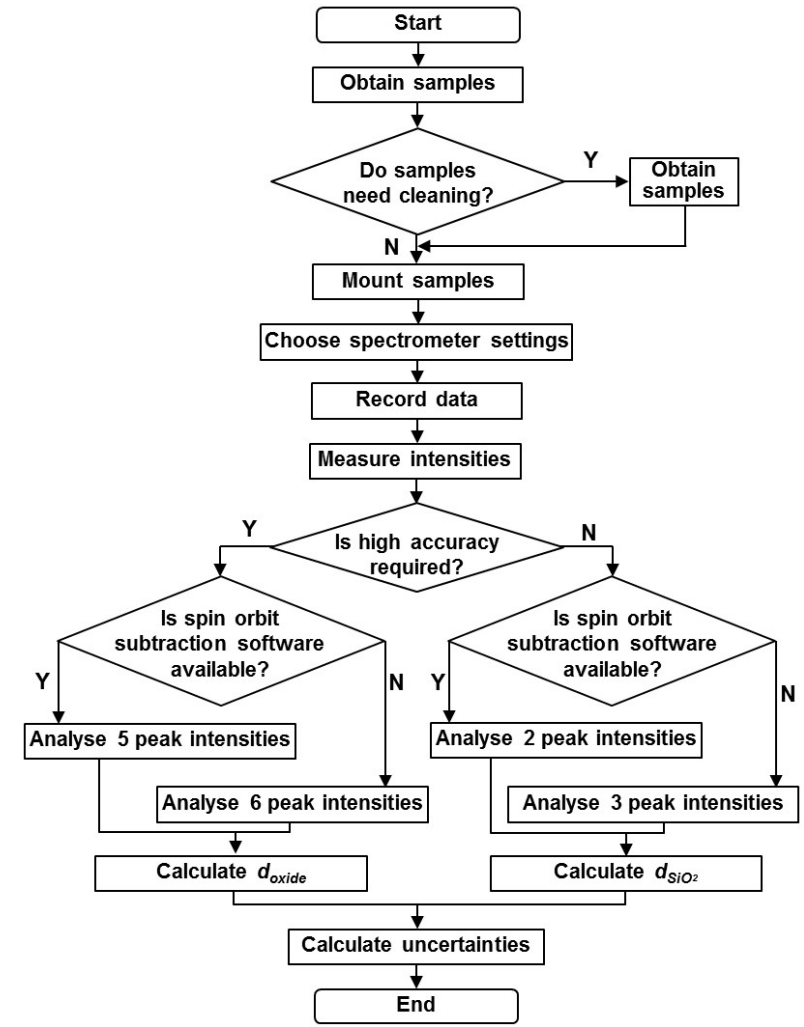

Figure 3. Flowchart of the measurement procedure for the thickness measurement of $\mathrm{nm} \mathrm{SiO}_{2}$ films on $\mathrm{Si}(100)$ and $\mathrm{Si}$ (111) substrates by XPS ( $\mathrm{Y}$ and $\mathrm{N}$ at decision points are the usual "yes" and "no," respectively.)

ISO-14701 was published as a standard for the thickness measurement of $\mathrm{nm} \mathrm{SiO}_{2}$ films by XPS [19]. It was investigated in SC7 (XPS) and organized by Dr. Martin Seah of NPL from the measurement protocol of K-32. Figure 3 shows the flowchart of the measurement procedure. It includes detailed processes for sample cleaning, sample mounting, spectrometer settings, recording data, intensity measurements, oxide thickness calculations and for the determination of measurement uncertainties.

\subsection{Activities of KRISS in ISO/TC-201}

KRISS has participated as a $\mathrm{P}$ member in ISO/TC-201 since 1994. KRISS has proposed five new work item proposals, SC4, SC6 and SC7, as shown in Table 6.

ISO-20341, published in 2003, specifies a method by which to estimate depth resolution parameters. The SIMS depth profile of a delta-layer film shall be described using an exponential rising edge, a Gaussian-like rounded top and an exponential trailing edge. The depth resolution parameters, such as the leading edge decay length $\left(\lambda_{\mathrm{L}}\right)$, the Gaussian broadening $(\rho)$ and the trailing
Table 6. Current status of the new work item proposals proposed by KRISS.

\begin{tabular}{|c|c|c|}
\hline Title & $\mathrm{SC}$ & $\begin{array}{l}\text { Current } \\
\text { status }\end{array}$ \\
\hline $\begin{array}{l}\text { Method for estimating depth resolution pa- } \\
\text { rameters with multiple delta-layer reference } \\
\text { materials }\end{array}$ & SC6 & $\begin{array}{l}\text { ISO } \\
20341\end{array}$ \\
\hline $\begin{array}{l}\text { Determination of the surface normal for an- } \\
\text { gle-resolved XPS }\end{array}$ & SC7 & stop \\
\hline $\begin{array}{l}\text { Measurement of sputtering rates using multi- } \\
\text { layered thin films }\end{array}$ & $\mathrm{SC} 4$ & $\mathrm{CD}$ \\
\hline $\begin{array}{l}\text { Determination of the interface location and } \\
\text { layer thickness in the SIMS depth profiling of } \\
\text { multilayers }\end{array}$ & 6 & WD \\
\hline $\begin{array}{l}\text { Compositional depth profiling of mul- } \\
\text { ti-element alloy films by surface analysis } \\
\text { methods using CRMs }\end{array}$ & SC4 & NWIP \\
\hline
\end{tabular}

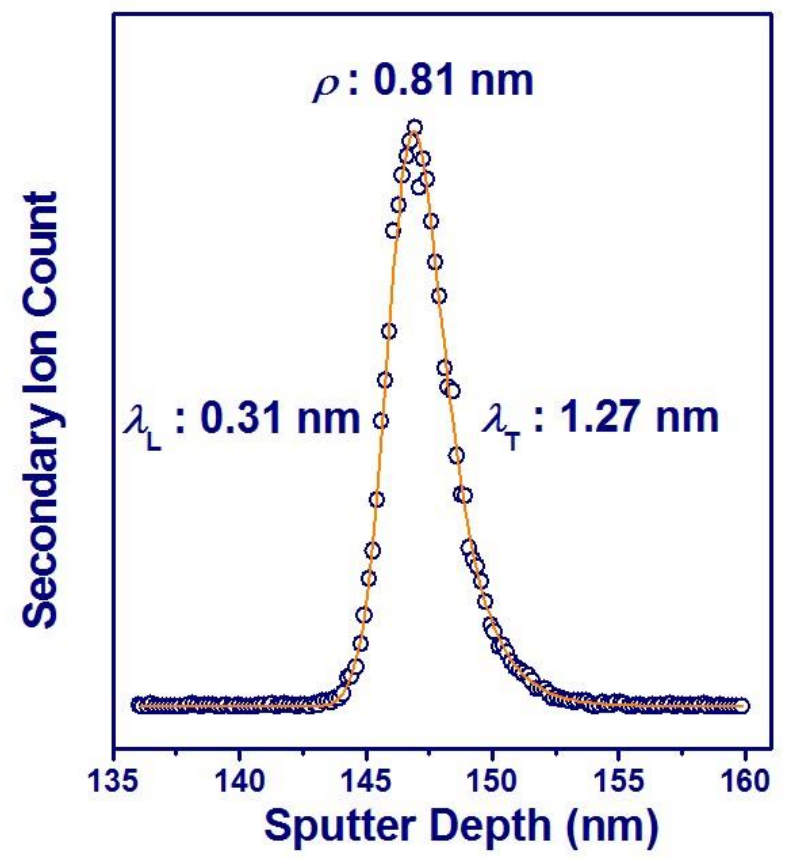

Figure 4. An example to estimate the depth resolution parameters from the depth profile of a delta-layer film. (color online)

edge decay length $\left(\lambda_{\mathrm{T}}\right)$, can be determined by deconvolution of the profile, as shown in Figure 4 [20,21].

The second proposal, entitled "Determination of the surface normal for angle-resolved XPS" was halted due to experimental difficulty in realizing the effect of a solid angle. Although two methods have published, more systematic studies are still required.[10,22] The other three proposals are being investigated. 


\section{Certified Reference Materials}

The third parameter of the standardization is certified reference materials (CRM). CRM can be a practical way to disseminate the measurement traceability of national metrology institutes. The measurand of CRM should be certified by traceable methods based on CMCs. For this reason, CRMs are generally developed by NMIs such as National Institute of Standards and Technology (NIST, USA), the National Physical Laboratories (NPL, UK), Bundesanstalt für Materialforschung und-Prüfung (BAM, Germany), the National Metrology Institute of Japan (NMIJ, Japan), and KRISS.

NIST SRM-2133, 2134 and 2137 are ion- implanted Si CRMs for the calibration of concentrations by the determination of the relative sensitivity factors of $\mathrm{P}$, As and $\mathrm{B}$ in $\mathrm{Si}$ (ISO-18114) [23]. NIST SRM-2137 can be used for the depth profiling of boron in silicon (ISO-14237 and ISO-17560). NIST SRM-2134 can be used for the depth profiling of arsenic in silicon (ISO-12406). NIST SRM-2135c is a Ni/Cr multilayer film for depth profiling analysis.

NPL has developed an organic multilayer reference material (OML) to establish the depth scale, depth resolution, sensitivity and linearity of the response for analysis methods [24]. Copper, silver and gold reference materials are disseminated for calibrating energy scales in XPS and AES, as described in ISO-15472, ISO 17973 and ISO-17974.

BAM-L200 is a nanoscale stripe pattern for the testing of lateral resolutions and calibration of length scales during elemental mapping by SIMS, AES, XPS and EDX [25]. It can be used by all surface analysis methods which are sensitive to a material contrast between $\mathrm{Al}_{0.7}$ $\mathrm{Ga}_{0.3} \mathrm{As}$ and GaAs.

NMIJ CRM 5203-a is a GaAs/AlAs super-lattice film fabricated by molecular beam epitaxy [26]. It was developed for use in controlling the precision of the analysis or for adjusting the measurement conditions during the depth profiling analysis by ion sputtering with AES, XPS and SIMS. This CRM can be used as a reference material for optimization using the layered systems described in ISO-14606.

KRISS has developed various types of thin-film CRMs for practical surface analysis by an ion beam sputtering deposition system.[27] An ion beam sputter deposition chamber is connected to XPS for the in-situ characterization of the surface chemical state and surface compositions. With this combined target system, various types of thin films, such as pure metal, oxide, multilayer and binary alloy films, can be grown.

\subsection{Multilayer Films for Depth Profiling}

Multilayer thin-film CRMs can be used to calibrate the depth scale, to evaluate the depth resolution parameters and to optimize the sputtering conditions so as to mini-

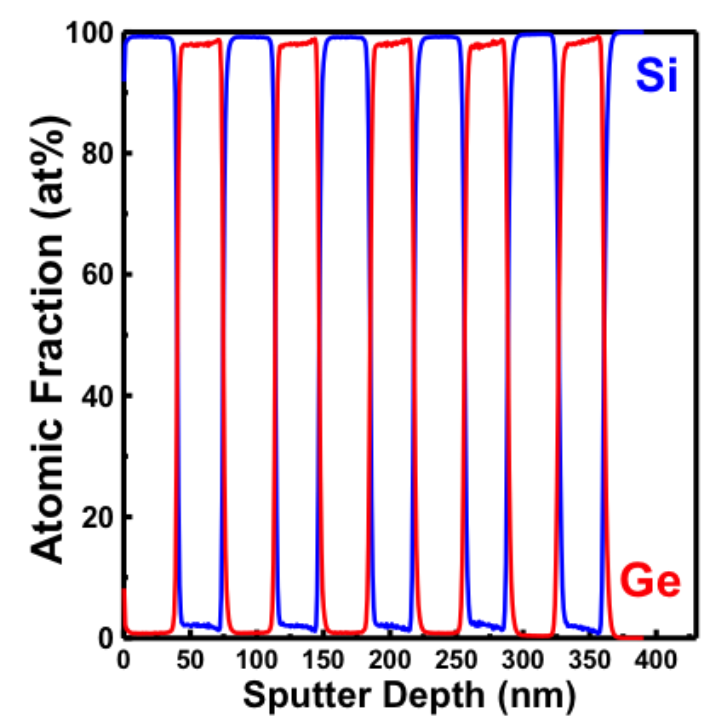

Figure 5. A compositional SIMS depth profile of a Si/Ge multilayer thin-film CRM by a $1 \mathrm{keV}$ oxygen ion beam. (color online)

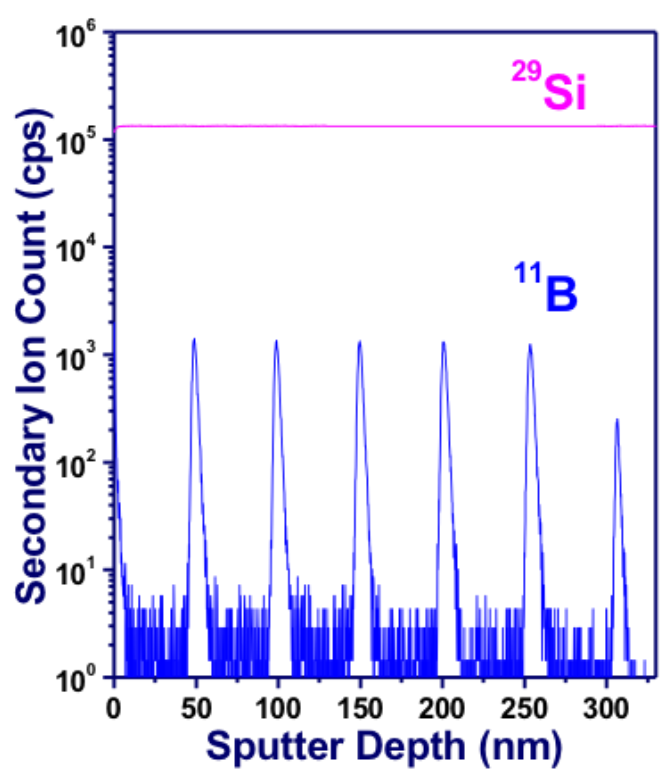

Figure 6. A SIMS depth profile of a Si/B-doped Si multiple deltalayer thin-film CRM. (color online) 
mize the depth resolution during sputter depth profiling by XPS, AES and SIMS. The thickness of multilayer thin-film CRMs grown on Si (100) wafers can be certified by high-resolution TEM from the lattice constant of the crystalline Si substrate. However, a line scan by scanning TEM/EDX will be helpful for the reliable certification of the layer thicknesses via clear determination of the interfaces.

$\mathrm{Ta} / \mathrm{Ta}_{2} \mathrm{O}_{5}$ multilayer and $\mathrm{Si} / \mathrm{Ge}$ multilayer thin-film CRMs have developed. These films can be used to optimize the sputtering conditions during depth profiling, as described in ISO/TR-15969 [28]. Si/Ge multilayer thin-film CRMs can also be used for measurements of the sputtering rate and for determinations of the interface location and layer thickness in the SIMS depth profiling of multilayers [29]. Figure 5 shows a compositional SIMS depth profile of a Si/Ge multilayer thin film.

The raw profile was converted to a compositional SIMS depth profile by the RSFs as determined from an alloy reference film. The locations of the interfaces can be clearly seen, and the layer thicknesses can also be determined correctly.

Multiple deltalayer thin-film CRMs of Si/B-doped Si, $\mathrm{Si} / \mathrm{GaAs}$ and $\mathrm{Si} / \mathrm{Ge}$ can be used for the determination of depth resolutions. Figure 6 shows a SIMS depth profile of a Si/B-doped Si multiple deltalayer thin-film CRM. This specimen can be used to estimate the depth resolution parameters as described in ISO-20341 [30]. The $\mathrm{Si} / \mathrm{Ge}$ multiple deltalayer thin-film CRM can be used to calibrate the SIMS depth scale by calibration of the step-height scale of a profilometer to measure the crater depth in a SIMS depth profiling analysis [31].

\subsection{Doped CRMs for Quantification by SIMS}

Although a quantitative analysis by SIMS is difficult due to the severe matrix effect, it is feasible as a means of quantitative analysis of minor impurities, where the matrix effect can be ignored. Ion-implanted CRMs with a low atomic concentration are generally used for SIMS quantification because choosing the doping elements and substrate materials is easy. However, the SIMS quantification process using an ion-implanted reference material is somewhat complicated because the in-depth distribution of the implanted element is not uniform. The entire range of the implanted zone must be profiled to determine the total number of the implanted atoms, and the sputtered depth should be precisely determined directly or indirectly by a profilometer to estimate the analyzed volume.

For the simple quantification of doping elements, a new method was suggested. If the atomic concentration of the doping element is uniform with the depth, the concentration of the element can be simply derived from the relative ratio of the element and the matrix element. ISO-14237 describes how to calculate the atomic concentration of B on a Si substrate using a uniformly doped $\mathrm{Si}$ specimen as a secondary reference material, commonly used in normal analyses. The atomic concentration of B in the uniformly doped $\mathrm{Si}$ specimen can be certified by an ion-implanted CRM. NIST SRM 2137 is the only CRM used as a primary reference.

KRISS developed a B-doped Si thin-film CRM as a reference material for the SIMS quantification of $B$ in silicon. It was fabricated by ion-beam sputtering deposition. B is uniformly doped with the depth in the Si thin film by the co-sputtering of $\mathrm{B}$ and $\mathrm{Si}$ using a composite target. The atomic concentration of $\mathrm{B}$ in $\mathrm{Si}$ was certified from the relative sensitivity factor of B determined from NIST SRM 2137.

Figure 7 shows a SIMS depth profile of the B-doped Si thin film developed using an $\mathrm{O}_{2}{ }^{+}$ion beam. The inten-

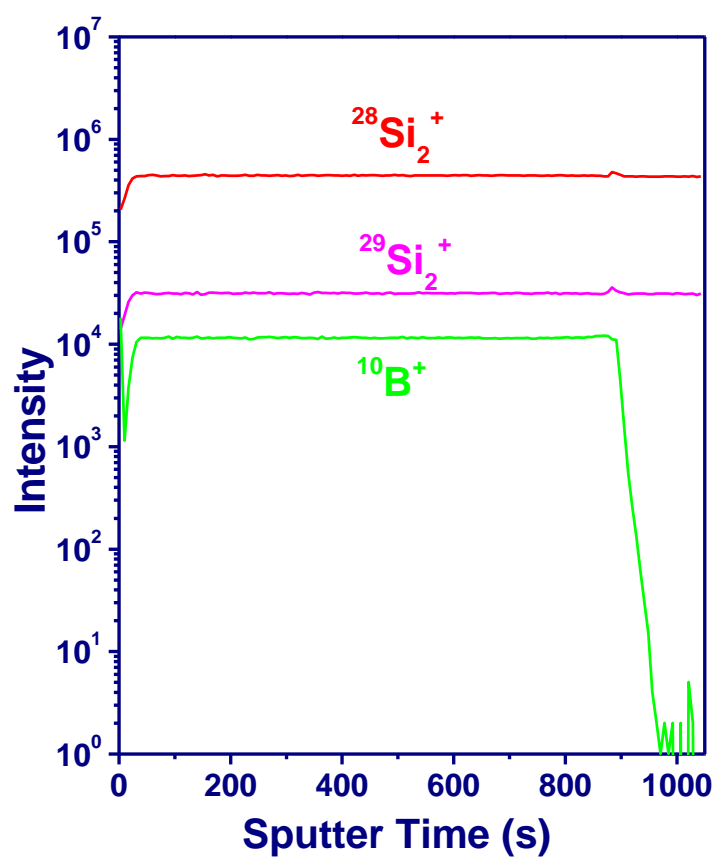

Figure 7. A SIMS depth profile of a B doped Si thin film by $\mathrm{O}_{2}{ }^{+}$ion beam. (color online) 
sities of ${ }^{28} \mathrm{Si}_{2}{ }^{+},{ }^{29} \mathrm{Si}_{2}{ }^{+}$and ${ }^{10} \mathrm{~B}^{+}$are uniform with the depth. In this CRM, the relative sensitivity factor of $\mathrm{B}$ can be easily determined from the relative intensities of $\mathrm{B}$ and the Si matrix. The RSF can be obtained at any point of the SIMS depth profile because the intensities of B and $\mathrm{Si}$ are constant with the depth. The uniformly doped $\mathrm{Si}$ thin film can also be used as a reference material to calibrate the depth scale because the interface is very clear.

\subsection{Alloy Thin-film CRMs for Quantification}

Alloy thin-film CRMs have also been developed by KRISS by means of ion-beam sputtering deposition. During the growth of the $\mathrm{AB}$ alloy films, the relative atomic fractions of the two elements can be controlled by the co-sputtering of A and B targets. The atomic fractions can be monitored by in-situ XPS analysis. The compositions of the $A B$ alloy films should be certified by ID-ICP/MS.

Fe-Ni alloy films (KRISS CRM103-04-012) were used as reference materials for pilot study P-98 and in key comparison K-67. Figure 8 shows the AES spectra of pure $\mathrm{Fe}$, pure $\mathrm{Ni}$ and three $\mathrm{Fe}-\mathrm{Ni}$ alloy films.

\section{Conclusions}

For the practical applications of surface analysis methods in advanced industries, these methods should be

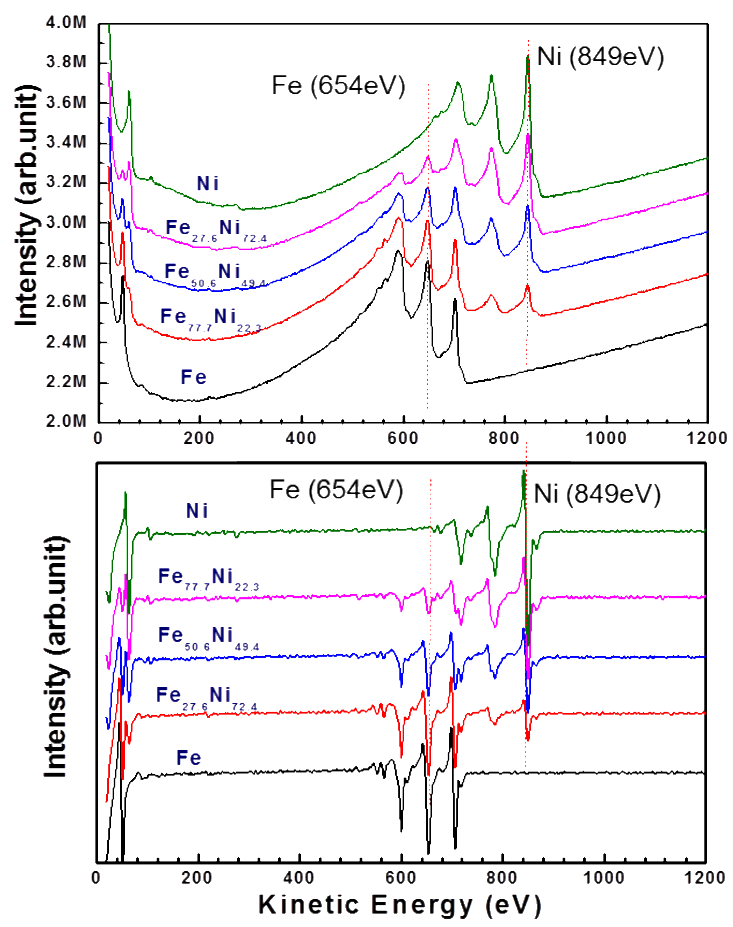

Figure 8. AES spectra of pure $\mathrm{Fe}$, pure $\mathrm{Ni}$ and three $\mathrm{Fe}-\mathrm{Ni}$ alloy films. (color online) standardized. The key parameters for standardization, i.e., measurement traceability, measurement procedure and certified reference material, should be prepared to improve the measurement reliability.

The measurement traceability of surface analysis methods is investigated by CCQM SAWG via pilot studies and key comparisons. National metrology institutes can obtain the right to certify a reference material by claiming the calibration and measurement capabilities for the metrological issue. Two key comparisons for the thickness measurement of $\mathrm{nm} \mathrm{SiO}_{2}$ films (K-32) and for the analysis of the atomic fractions of $\mathrm{Fe}-\mathrm{Ni}$ alloy films (K-67) have been performed. KRISS has organized two pilot studies (K-98 and K-140) and two key comparisons (K-67 and K-129). As a result of the two KCs, KRISS claimed two CMCs for the thickness measurement of nm $\mathrm{SiO}_{2}$ films and for the analysis of the atomic fractions of Fe-Ni alloy films.

International standards for surface chemical analysis are developed by ISO/TC-201. Since 1991, 59 international standards have been developed in the area of surface chemical analysis by nine subcommittees. KRISS has participated in ISO/TC-201 as a P member and has contributed to the development of various international standards. KRISS suggested five new work item proposals.

NMIs develop certified reference materials as a practical way to disseminate the measurement traceability of NMIs. NIST, NPL, BAM and NMIJ have developed various types of CRMs. KRISS has developed three types of thin-film CRMs. These CRMs are used to establish measurement traceability and to develop international standards. The most important application of a CRM is to serve as a standard measurement system during the fabrication processes used by advanced industries.

\section{References}

[1] http://www.bipm.org/en/about-us/.

[2] M. P. Seah, S. J. Spencer, F. Bensebaa, I. Vickridge, H. Danzebrink, M. Krumrey, T. Gross, W. Oesterle, E. Wendler, B. Rheinländer, Y. Azuma, I. Kojima, N. Suzuki, M. Suzuki, S. Tanuma, D. W. Moon, H. J. Lee, H. M. Cho, H. Y. Chen, A. T. S. Wee, T. Osipowicz, J. S. Pan, W. A. Jordaan, R. Hauert, U. Klotz, C. van der Marel, M. Verheijen, Y. Tamminga, C. Jeynes, P. Bailey, S. Biswas, U. Falke, N. 
V. Nguyen, D. Chandler-Horowitz, J. R. Ehrstein, D. Muller and J. A. Dura, Surf. Interface Anal., 36, 1269 (2004).

[3] M. P. Seah, Metrologia, 45, Tech. Suppl., 08013 (2008).

[4] M. P. Seah, W. E. S. Unger, H. Wang, W. Jordaan, Th. Gross, J. A. Dura, D. W. Moon, P. Totarong, M. Krumrey, R. Hauert and M. Zhiqiang, Surf. Interface Anal., 41, 430 (2009).

[5] K. J. Kim, Metrologia, 46, Tech. Supp., 08006 (2009).

[6] K. J. Kim, Metrologia, 47, Tech. Supp., 08011 (2010)

[7] "Summary of CCQM Key Comparisons and Pilot Studies" http://www.bipm.org/en/committees/cc/cc qm/pilot-cc.html.

[8] K. J. Kim, Metrologia, 52, Tech. Supp., to be published (2015).

[9] K. J. Kim, Y. S. Kim, J. S. Jang, J. W. Kim, K. W. Kim, Metrologia, 45, 507 (2008).

[10] K. J. Kim, J. S. Jang and D. W. Moon, Metrologia, 43, L28 (2006).

[11] J. T. Grant, P. Williams, J. Fine and C. J. Powell, Surf. Interface Anal., 13, 46 (1988).

[12] K. J. Kim, D. W. Moon, C. J. Park, D. Simons, G. Gillen, H. Jin and H. J. Kang, Surf. Interface Anal., 39, 665 (2007).

[13] D. Abou-Ras, R. Caballero, C-H. Fischer, C. A. Kaufmann, I. Lauermann, R. Mainz, H. Mönig, A. Schöpke, C. Stephan, C. Streeck, S. Schorr, A. Eicke, M. Döbeli, B. Gade, J. Hinrichs, T. Nunney, H. Dijkstra, V. Hoffmann, D. Klemm, V. Efimova, A. Bergmaier, G. Dollinger, T. Wirth, W. Unger, A. A. Rockett, A. Perez-Rodriguez, J. Alvarez-Garcia, V. Izquierdo-Roca, T. Schmid, P.-P. Choi, M. Müller, F. Bertram, J. Christen, H. Khatri, R. W. Collins, S. Marsillac, and I. Kötschau, Microsc. Microanal., 17, 728 (2011).

[14] C. L. Perkins, B. Egaas, I. Repins and B. To, Appl. Surf. Sci., 257, 878 (2010).

[15] M. Topič, F. Smole and J. Furlan, J. Appl. Phys., 79, 8537 (1996).

[16] A. Eicke, S. Spiering, A. Dresel and M. Powalla, Surf. Interface Anal., 40, 830 (2008).

[17] J. S. Jang, H. H. Hwang, H. J. Kang, J. K. Suh, H. S. Min, M. S. Han, K. H. Cho, Y. D. Chung, D. H.
Cho, J. Kim and K. J. Kim, Metrologia, 49, 522 (2012).

[18] http://www.iso.org/iso/home.htm.

[19] M. P. Seah, Surf. Interface Anal., 44, 876 (2012).

[20] M. G. Dowsett, G. Rowland., P. N. Allen and R. D. Barlow, Surf. Interface Anal., 21, 310 (1994).

[21] D. W. Moon, J. Y. Won, K. J. Kim, H. J. Kim, H. J. Kang, M. Petavic, Surf. Interface Anal., 29, 362 (2000).

[22] M. P. Seah and S. J. Spencer, Surf. Interface Anal., 33, 640 (2002).

[23] http://www.nist.gov/srm/

[24] http://www.npl.co.uk/science-technology/surfaceand-nanoanalysis/services/

[25] http://www.rm-certificates.bam.de/en/certificates

[26] https://www.nmij.jp/english/service/C/crm/

[27] Catalogue: http://www.kriss.re.kr/eng/file/2014121 5crm.pdf, Detailed information: kjkim@kriss.re.kr

[28] S. Hofmann, Surf. Interface Anal., 33, 453 (2002).

[29] K. J. Kim, J. S. Jang, D. W. Moon, and H. J. Kang, Metrologia, 47, 253 (2010).

[30] D. W. Moon, Surf. Interface Anal., 37, 646 (2005).

[31] K. J. Kim, D. W. Moon, P. Chi, and D. Simons, Surf. Interface Anal., 37, 802 (2005). 


\section{Discussion and Q\&A with the reviewers}

The figures and page numbers referred to below indicate those in the original manuscript.

\section{Reviewer 1. Kazuhiro Yoshihara (Scienta Omicron,} Inc.)

This is a fine and very informative review on the standardization activity of KRISS, and should be published without any revision. Especially, CCQM activities are clearly summarized. We can understand CCQM is the important activity to ensure the traceability, and CRMs play an important role.

\section{[Q1_1]}

Is it possible to get CRMs for a non-member of SAWG of CCQM?

[A1_1]

Yes it is possible to get CRMs by a non-member of SAWG of CCQM. However, participation in key comparison is limited to the members of SAWG of CCQM.

\section{[Q1_2]}

If possible, please indicate the protocol or procedure to obtain CRMs from KRISS in this paper.

\section{[A1_2]}

Although the CRMs can be obtained by Korean people from the Korean website of KRISS, there is no way to get the KRISS CRMs in the English website of KRISS. The website to get a CRM catalogue (http://www.kriss.re.kr/eng/file/20141215crm.pdf) and an e-mail address to get the detailed information about CRM (kjkim@kriss.re.kr) were described in reference [27].

\section{Reviewer 2. Takaharu Nagatomi (Asahi Kasei Cor-} poration)

\section{[Q2_1]}

Page 3, first paragraph, "because the thickness of $\mathrm{SiO}_{2}$ film by XPS is determined....".

Of course, the offset value is one of the important parameter for quantification and I agree with that the offset for XPS will be zero since the intensity of $\mathrm{Si}^{4+}$ component is used. However, this point is not a proof showing the XPS is the most reliable technique, I think. For instance, the attenuation length is not constant for the wide range of the $\mathrm{SiO}_{2}$ thickness from 1.5 to $8 \mathrm{~nm}$. Would you add brief explanation why XPS is considered to be the most reliable technique?

[A2_1]

We have some data to show that the effective attenuation length (EAL) is constant irrespective of thickness. At first, the thicknesses of a series of thermally grown $\mathrm{SiO}_{2}$ films were measured by XPS and TEM. The thickness measured by XPS using the same EAL showed a good linear relationship with those measured by TEM. (Figure 4 in Metrologia, 45, 507 (2008))

A series of $\mathrm{SiO}_{2}$ films were deposited as a function of deposition time. The thicknesses were measured by in-situ XPS using the same EAL. It showed also a good linear relationship with the nominal thickness determined as a function of deposition time. (Figure 3 in Surf. Interface Anal., 39, 512 (2007))

These two data indicate that the EAL of photoelectron is constant or the variation of EAL is not so large. As a result, if we determine the EAL correctly, the thickness of $\mathrm{SiO}_{2}$ films can be determined reliably.

However, I do not want to include these sentences in the manuscript because some people believe that the attenuation length is not constant for the wide range of the $\mathrm{SiO}_{2}$ thickness from 1.5 to $8 \mathrm{~nm}$.

[Q2_1_2]

This point is very important. The above discussion should be added at the end of the manuscript as Q\&A during refereeing.

[A2_1_2]

Yes, this point is very important. However, in this paper, I just described the results of P-84 and K-32. I did not describe that the XPS is the most reliable technique for the thickness measurement. So, I do not want further discussions in this paper.

\section{[Q2_2]}

Page 3, last line of the first paragraph of "2.2. Quantification of Fe-Ni...", "In this case, the matrix effect and the surface compositional change by ion sputtering can be counterbalanced [12]."

I cannot figure out why matrix effect and preferential sputtering are counterbalanced each other. Would you please comment on that? 


\section{[A2_2]}

If the physical properties and chemical compositions are similar in a reference specimen and an analysis specimen, the matrix effect in the reference specimen and the analysis specimen may be the same because the atomic fractions of the constituent elements are very similar in the two specimens. From the same reason, the surface composition changes by ion beam sputtering should be also similar in the two specimens.

[Q2_2_2]

I think that the authors want to say that the matrix effect and preferential sputtering are counterbalanced between a reference and a sample to be measured. The expression in the paper seems that they are counterbalanced each other. The expression should be revised.

\section{[A2_2_2]}

$\mathrm{OK}$, it was revised to "In this case, the matrix effect and preferential sputtering are counterbalanced between a reference and a sample to be measured [12]."

\section{[Q2_3]}

Page 3, second paragraph, "ARRSF"

ARRSF is not common. The definition should be described for understanding of readers.

\section{[A2_3]}

The sentence "The second type is alloy reference RSF (ARRSF) which is determined from the certified atomic fractions of the reference film. The ARRSFs of $\mathrm{Fe}\left(\mathrm{R}_{\mathrm{Fe}}{ }^{\mathrm{AR}}\right)$ and $\mathrm{Ni}\left(\mathrm{R}_{\mathrm{Ni}}{ }^{\mathrm{AR}}\right)$ were derived from a certified Fe51-Ni49 alloy film by dividing the peak intensities Fe $\left(\mathrm{I}_{\mathrm{Fe}}{ }^{\mathrm{AR}}\right)$ and $\mathrm{Ni}\left(\mathrm{I}_{\mathrm{Ni}}{ }^{\mathrm{AR}}\right)$ by the certified atomic fractions of $\mathrm{Fe}\left(\mathrm{C}_{\mathrm{Fe}}{ }^{\mathrm{AR}}\right)$ and $\mathrm{Ni}\left(\mathrm{C}_{\mathrm{Ni}}{ }^{\mathrm{AR}}\right)$, respectively." was added.

\section{[Q2_3_2]}

The alloy reference RSF is basically the same as the atomic RSF defined for AES and XPS (please see ISO18118). What is the difference between them?

\section{[A2_3_2]}

Sorry, I do not agree with your opinion that the ARRSF is the same as the ARSF in ISO-18118. The ARSF ( $S_{i}^{A t}$ ) in ISO-18118 is defined by the following equation

$$
S_{i}^{A t}=\left(\frac{N^{k e y}}{N_{i}}\right) S_{i}^{E} .
$$

where $\mathrm{N}^{\mathrm{key}}$ and $\mathrm{N}_{\mathrm{i}}$ are the atomic densities for the key element and for element $\mathrm{i}$, respectively.

The considerations of the atomic densities in the
ARSFs and the matrix correction factors in the AMRSFs are the basic differences from the ARRSFs.

The atomic densities, matrix correction factors and sputtering effect correction are not required in the determination of the ARRSFs.

\section{[Q2_4]}

Page 3, 3rd paragraph,

The author intended to mention that ARRSF is better than PERSF. For me, it seems to make no meaning because PERSF will not give better atomic fraction. For this kind of discussion, at least, atomic RSF must be used according ISO18118 for AES and XPS. Would you please give a short explanation why ARSF was not employed in P-98?

[A2_4]

There was no reason. P-98 was just planned to compare the effects of the PERSFs and ARRSFs on the quantification of $\mathrm{Fe}-\mathrm{Ni}$ alloy films.

\section{[Q2_5]}

Page 4, first paragraph, "CIGS was selected..."

The homogeneity of composition of CIGS is not good. In addition, the control of the composition may be difficult. From these points of view, CIGS does not seem to be suitable as a reference sample for quantification. Is my understanding correct? If so, would you please give a comment why this material system was selected as a reference sample in $\mathrm{P}-140$ ?

[A2_5]

I agree with your opinion that CIGS is not suitable as a reference sample for the quantification because the atomic fractions are not homogeneous with depth and it is a multi-element alloy film with four elements and rough surface. So it can be said to be the worst case for the quantitative analysis.

However, if the quantitative results can be derived from the worst system, the method can be widely applied for the quantification of multi-element alloy films.

The uncertainty in the quantification of CIGS films in $\mathrm{K}-129$ is much better than that of the quantification of $\mathrm{Fe}-\mathrm{Ni}$ alloy film in K-67. (This result will be published soon)

\section{[Q2_5_2]}

The concept for the reference sample does not seem to be reasonable for me. The error bar in the composition of 
reference samples should be small as much as possible. The homogeneity is also required. Would you please comment on this point?

\section{[A2_5_2]}

I agree with your opinion that the error bar in the composition of reference samples should be small and homogeneous with depth. However, if the inhomogeneous sample can be also used as reference sample using a special method, it will be very useful. The possibility of the inhomogeneous reference sample was confirmed in the pilot study P-140.

\section{[Q2_5_3]}

In the reply, the author showed that the uncertainty in $\mathrm{K}-129$ is smaller than that of $\mathrm{K}-67$. What is the main reason of this? Is that measurement condition? Please comment on this point as well.

\section{[A2_5_3]}

The uncertainties of key comparison reference value (KCRV) of K-67 and K-129 are 1.23 at\% and 0.36 at\%, respectively. It means that the standard deviation of the submitted atomic fractions is the main reason of the large difference of uncertainty.

\section{[Q2_6]}

Page 5,top paragraph in the right column and last paragraph, "TNC method was recommended / TNC method is reliable..."

As a comparison of the quantification, the quantification should be performed according to ISO18118. TNC method required to depth profile whole depth of reference film and ICP/MS. This is not practical method. In addition, the basic idea of this technique is the same as conventional way described in ISO 18118, in which RSF is used. I cannot understand what is a merit of this method. I would like to ask authors to give a comment on this point.

\section{[A2_6]}

ISO-18118 was developed for the quantitative analysis of homogeneous materials by AES and XPS. So it is not suitable to non-homogeneous multi-element alloy films.

The ARRSFs determined from the reference alloy films using TNC method are different from the ARSFs and AMRSFs in ISO-18118.

The strongest merit of TNC method is the quantification of multi-element alloy films with non-homogeneous depth distribution.

\section{[Q2_6_2]}

ISO 18118 can be applied to multi-elemental system. In addition, the basic idea of ARRSF is the same as the ARSF. Furthermore, if the quantification described here is applied to the sample with high accuracy, the homogeneity of the sample is required since the composition of the sample should be similar to the reference as the authors mentioned. We can apply the ISO18118 to a sample with inhomogeneous elemental depth distribution in the same way as the authors have done in the paper. We can obtain the similar results. So, I cannot still understand the advantage of the method described in this paper.

\section{[A2_6_2]}

Yes, ISO 18118 can be applied to multi-elemental system. However, I think the application of ISO-18118 for the quantification of multi-element alloy films such as CIGS films will be very complicated because so many theoretical parameters should be applied to determine the AMRSFs.

Do you have any example about the quantification of multi-element alloy films (with more than three elements) using ISO-18118? If you provide the example, it will be very useful for the quantification of multi-element alloy films.

\section{[Q2_6_3]}

To the best of our knowledge, there is no good example about the quantification of multi-element alloy films (with more than three elements) using ISO-18118. 